\title{
Herpes zoster infection after an uncomplicated cataract surgery: A case report
}

\author{
Nursen Oncel Acir ${ }^{1}$, Hamiyet Solmaz ${ }^{1}$, Servet Cetinkaya², Cemil Savas ${ }^{1}$, Zeynep Dadaci ${ }^{1}$, Mehmet Borazan ${ }^{1}$
}

\begin{abstract}
ABST RACT
Herpes zoster is an acute viral infection characterized by painful dermatomal vesicular eruption on an erythematous base. It results from reactivation of latent varicella zoster virus infection that has persisted in dorsal root ganglia. The disease is commonly encountered in elderly and immunocompromised patients. In the current report, we have presented a 70-year-old woman who developed herpes zoster infection on her left subpectoral-dorsal site following uncomplicated cataract surgery. The patient was consulted with dermatology clinic and treated successfully.
\end{abstract}

Keywords: herpes zoster, cataract surgery, zona

\section{INTRODUCTION}

Herpes zoster $(\mathrm{HZ})$, also known as shingles, is the result of reactivation of endogenous latent varicella zoster virus (VZV), which is in dormant stage within sensory dorsal root, cranial nerve, and autonomic ganglia (1). HZ can manifest any time after a primary infection with VZV (i.e., varicella or chickenpox). The activated virus travels back down the corresponding cutaneous nerve to the adjacent skin, causing typically a painful, unilateral vesicular eruption in a restricted dermatomal distribution (2). The diagnosis of $\mathrm{HZ}$ is mainly made clinically, based on the distinctive clinical appearance and symptomatology. Laboratory tests are not usually necessary unless the rash is atypical (1).

It is well known that the incidence and severity of $\mathrm{HZ}$ infection increases with age and in people with depressed immunity (2). Besides, some triggering factors such as emotional stress, malignancy, presence of chronic illness, use of steroids and immunosuppressive agents, radiation, cryotherapy, liver biopsy, axillar nerve block, spinal surgery, chemical or actinic stimuli, massage and laser therapy were reported previously $(1,3)$

The aim of this study is to present a patient who developed acute $\mathrm{HZ}$ infection following cataract surgery. To the best of our knowledge, this is the first case report presented systemic $\mathrm{HZ}$ infection manifestation following cataract surgery.

\section{CASE PRESENTATION}

A 70-year-old woman admitted to the ophthalmology department with the complaint of decreased visual acuity on her left eye for 5 months. She had controlled diabetes mellitus for 10 years in her medical history. The ophthalmic examination revealed senile nuclear cataract in her left eye and she was offered cataract surgery. There were no pathologic changes in her laboratory findings preoperatively and she underwent an uncomplicated cataract surgery. She was prescribed frequent use topical steroid and antibiotic. On the $3^{\text {rd }}$ day of the postoperative period, she admitted to ophthalmology clinic for the first visit. She has no complaint in her operated eye. In her

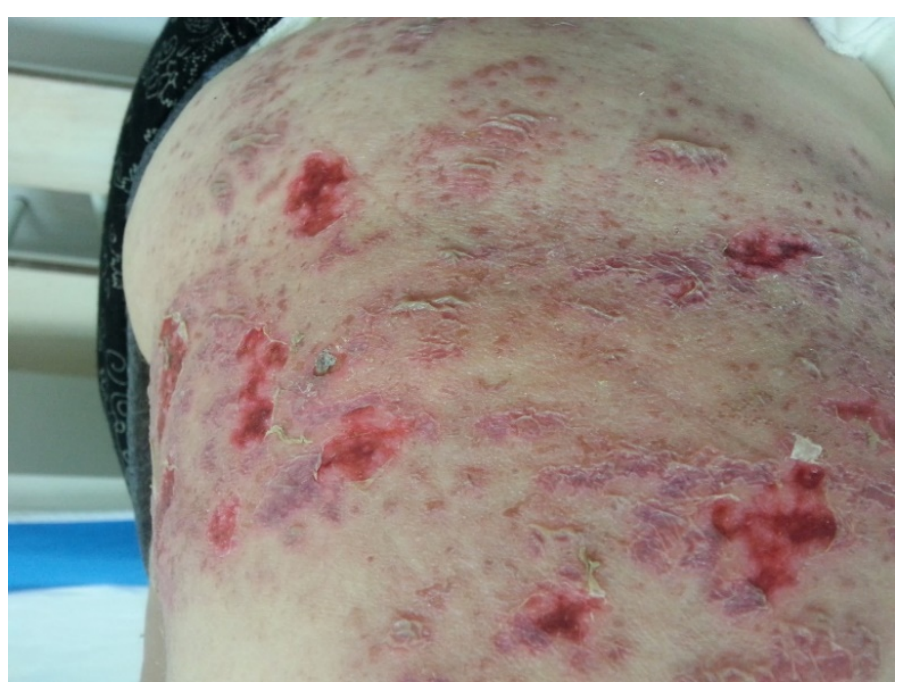

Figure 1: The typical vesicular rash of herpes zoster can be seen at the left lumbosacral site of the patient

ophthalmologic examination there were no pathologic findings about surgery and all findings were consistent with normal postoperative process. But the patient stated that she felt sick and weak and had pain and red skin lesions on her left trunk. Physical examination revealed erythematous skin, multiple herpetiform and hemorrhagic vesicles on left dorsal lumbar site (Figure 1).

As a result of the consultation with dermatology department, she was diagnosed as $\mathrm{HZ}$ infection. The patient treated with oral acyclovir and topical analgesics, the lesions almost healed and the pain resolved within one month. There was no eye involvement in this process.

\section{DISCUSSION}

We reported an elderly diabetic patient who had $\mathrm{HZ}$ infection following cataract surgery. The occurrence of

\footnotetext{
1 Konya, Turkey

Turkish Red Crescent Hospital, Department of Ophthalmology, Konya, Turkey
}

Received: 29 J uly 2015, Accepted: 10 Sep 2015
Correspondence: Nursen Oncel Acir, MD

Meram, Konya, Turkey

Phone: +903323215242

Fax: +903323229410 
periocular $\mathrm{HZ}$ infection in patients with diabetes mellitus following cataract surgery has been reported in the literature $(4,5)$. While one report described the occurrence of infection is at ipsilateral V2 dermatome, another one defined it at contralateral dermatome. In both reports, infection site corresponds to periocular region. So, reporters suggested that combination of phacoemulsification surgery and presence of diabetes mellitus caused a locally weakened immunological situation. However, in the current report, the infection site of our patient is not periocular region, it is at lumbosacral region. But our patient had no any immunodeficient condition except diabetes mellitus, similar with those reports. In a study, it has been described that the incidence of $\mathrm{HZ}$ among individuals with diabetes was higher than among those without (6). So, the presence of the diabetes in our patient may be considered as a risk factor for occurrence of the $\mathrm{HZ}$ infection.

In addition, exposure to emotionally stressful situations predisposes to $\mathrm{HZ}$ infection (1) and it's well known that undergoing a surgery is a serious stress factor even though it was performed safely with utmost care. So, we believe that, the relatively high level of anxiety of our patient preoperatively may contribute the occurrence of $\mathrm{HZ}$ infection following the surgery.

\section{REFERENCES}

1. Hata A, Kuniyoshi M, Ohkusa Y. Risk of Herpes zoster in patients with underlying diseases: a retrospective hospital-based cohort study. Infection. 2011;39(6):53744.

2. Oxman MN. Herpes zoster pathogenesis and cellmediated immunity and immunosenescence. J Am Osteopath Assoc. 2009; 109(6 Suppl 2):S13-7.

3. Lee MR, Ryman W. Herpes zoster following cryosurgery. Australas J Dermatol. 2005; 46(1):42-3.

4. Korber A, Franckson T, Grabbe S, Dissemond J. Ambilateral reactivation of herpes zoster V2 following cataract operation of both eyes. J Eur Acad Dermatol Venereol. 2007;21(5):712-3.
Another possible reason may be the frequent use of topical steroid drops in the postoperative period. Ocular steroids can be available in the systemic circulation and can cause systemic adverse effects. In a study, when the identical doses of steroid were administrated intravenously, intranasally and topically in the conjunctiva, the rates of systemic absorption have been found similar (7). Also, it has been previously reported that topical steroid eye drops may trigger the manifestation of $\mathrm{HZ}$ infection (8). So, we may speculate that, using topical steroid drops may possibly contribute to the development of $\mathrm{HZ}$ infection in our patient.

\section{CONCLUSION}

To sum up, for the patient presented in our report, it may be concluded that the presence of diabetes, surgical stress, and using topical steroid drops resulted in the development of $\mathrm{HZ}$ infection. Thus, when elderly and/or diabetic patients with cataract are operated, surgeons should be aware of the unexpected systemic symptoms and should observe the patients carefully in postoperative period.

5. Walland MJ. Presumed ophthalmic herpes zoster after contralateral cataract extraction. Acta Ophthalmol Scand. 1995; 1: 83-85.

6. Suaya J A, Chen SY, Li Q, Burstin SJ, Levin MJ . Incidence of herpes zoster and persistent post-zoster pain in adults with or without diabetes in the United States. Open Forum Infect Dis. 2014 2;1(2): ofu049.

7. Sigurdsson HH, Konráethsdóttir $F$, Loftsson $T$, Stefánsson E. Topical and systemic absorption in delivery of dexamethasone to the anterior and posterior segments of the eye. ActaOphthalmol Scand. 2007;85(6):598-602.

8. Farooq U, Dallol B. Acute confusion secondary to steroid eye drops. BMJ Case Rep. 2014; 24:2014. 